\title{
New near-infrared observations and lens-model constraints for UM673
}

\author{
E. Koptelova ${ }^{1,2,3}$, T. Chiueh ${ }^{1}$, W. P. Chen ${ }^{2}$, and H. H. Chan ${ }^{1}$ \\ ${ }^{1}$ Department of Physics, National Taiwan University, No.1, Sec. 4, Roosevelt Rd., 106 Taipei, Taiwan \\ e-mail: [ekaterina; chiuehth]@phys.ntu.edu.tw \\ 2 Graduate Institute of Astronomy, National Central University, Jhongli City, Taoyuan County 320, Taiwan \\ e-mail: wchen@astro.ncu.edu.tw \\ 3 Sternberg Astronomical Institute (SAI), Moscow M.V. Lomonosov State University, Universitetskii pr. 13, 119992 Moscow, Russia
}

Received 8 July 2013 / Accepted 1 April 2014

\begin{abstract}
Aims. We performed a detailed photometric analysis of the lensed system UM673 (Q0142-100) and an analysis of the tentative lens models.

Methods. High-resolution adaptive optics images of UM673 taken with the Subaru telescope in the $H$ band were examined. We also analysed the $J, H$ and $K$-band observational data of UM673 obtained with the $1.3 \mathrm{~m}$ telescope at the CTIO observatory.

Results. We present photometry of quasar components A and B of UM673, the lens, and the nearby bright galaxy using $H$-band observational data obtained with the Subaru telescope. Based on the CTIO observations of UM673, we also present $J$ - and $H$-band photometry and estimates of the $J, H$ and $K$-band flux ratios between the two UM673 components in recent epochs. The near-infrared fluxes of the A and B components of UM673 and their published optical fluxes are analysed to measure extinction properties of the lensing galaxy. We estimate the extinction-corrected flux ratio between components A and B to be about 2.14 mag. We discuss lens models for the UM673 system constrained with the positions of the UM673 components, their flux ratio, and the previously measured time delay.
\end{abstract}

Key words. gravitational lensing: strong - methods: data analysis - quasars: individual: UM673

\section{Introduction}

Foreground galaxies acting as strong lenses provide important information on the mass distribution on small scales. The mass can be constrained by relative positions, fluxes, and time delays between different components (images) of lensed quasars. When the lens mass model is known, the Hubble constant can be inferred from the time delays between the quasar images (Refsdal 1964). In many cases, however, the mass model of the lens is more complex than that of a single object. Keeton et al. (2000) predicted that at least $25 \%$ of lenses lie in environment (groups and clusters) that perturbs the lensing potential. The results of Treu et al. (2009), who found that approximately $20 \%$ of the lenses from their Sloan ACS Survey belong to known groups or clusters, agree well with the theoretical expectations. The galaxy groups are found in the observations of many gravitationally lensed quasars (see, e.g., Fassnacht \& Lubin 2002; Faure et al. 2004; Momcheva et al. 2006; Williams et al. 2006). Often, perturbing objects are detected on the line of sight towards lensed quasars (see, e.g., Tonry \& Kochanek 2000; Faure et al. 2004; Fassnacht et al. 2006; Momcheva et al. 2006). This line-of-sight mass might lead to an overestimation of the mass of the main lens (see, e.g., Wambsganss et al. 2005) and might have a substantial effect on lensing properties (Keeton 2003).

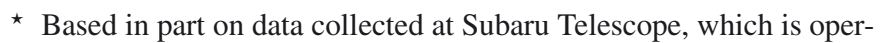
ated by the National Astronomical Observatory of Japan.

$\star \star$ The final reduced Subaru images presented in Figs. 1 and 2 are only available at the CDS via anonymous ftp to

cdsarc.u-strasbg.fr (130.79.128.5) or via

http://cdsarc.u-strasbg.fr/viz-bin/qcat?J/A+A/566/A36
Measured time delays and flux ratios between quasar images can help to assess properties of the perturbing mass and reduce uncertainties in the lens model (see, e.g., Keeton 2003; Keeton \& Moustakas 2008). Thus, MacLeod et al. (2009) have identified a secondary lensing galaxy through its effect on the fluxes of the lensed quasar H1413+117. (This second lens is associated with a high-redshift line-of-sight structure towards the quasar (Kneib et al. 1998).) For the same lensed system, Goicoechea \& Shalyapin (2010) used time-delay constraints to improve the model of the lens and to estimate the unknown redshift of the main lensing galaxy.

In this study we focus on the lensed system UM673 (Q0142100) discovered by Surdej et al. $(1987,1988)$. The system consists of images A and B (separated by 2.2") of a distant quasar at redshift $z_{\mathrm{q}}=2.719$ gravitationally lensed by an elliptical galaxy at redshift $z_{1}=0.49$ (Surdej et al. 1988; Smette et al. 1992; Eigenbrod et al. 2007). The fluxes of the two UM673 images are known to be very different, with image A being about seven times brighter than image $\mathrm{B}$. The time delay between images $\mathrm{A}$ and B of UM673 measured in Koptelova et al. (2012) and most recently in Oscoz et al. (2013) $(89 \pm 11$ and $72 \pm 22$ days, respectively) is shorter than expected from the singular isothermal ellipsoid model discussed in Keeton et al. (1998) and Lehár et al. (2000). Lehár et al. (2000) also estimated local tidal perturbations produced by nearby galaxies detected in the HST images of UM673. From their analysis, Keeton et al. (1998) and Lehár et al. (2000) concluded that the lens model requires an external shear to explain the positions of the UM673 images and their flux ratio. However, the orientation of the shear from modelling does not coincide with the direction to any of the nearby galaxies. 
Hence, new detailed observations of UM673 can be important for further understanding of the lens mass model.

The spectroscopic observations of UM673 show numerous mass concentrations on the line of sight to the quasar. Surdej et al. (1988) found the same absorbtion-line systems in the spectra of both components A and B (see also Smette et al. 1992 and Rauch et al. 2001). Most recently, Cooke et al. (2010) discovered a damped Ly $\alpha$ absorption (DLA) system at $z=1.63$ in the spectrum of component A of UM673. They also found a weak Ly $\alpha$ emission line in the spectrum of component B at the redshift of the DLA absorber.

The DLA absorber towards UM673 might indicate the existence of a massive object, a galaxy, as DLA systems are usually associated with the progenitors of normal spiral galaxies (Wolfe et al. 1986). Direct observations of the DLA galaxy could help to improve the lens model of UM673. Unfortunately, imaging of the DLA-associated galaxies is often complicated by their faintness and proximity to much brighter background quasars (see, e.g., Møller et al. 2002). As demonstrated by Chun et al. (2006, 2010) and Péroux et al. (2011), high-resolution deep adaptive optics (AO) observations offer a promising technique to achieve the necessary angular resolution and sensitivity to identify faint objects at small impact parameters from the line of sight to the bright sources.

With the goal of studying the UM673 close environment we conduct high-resolution AO imaging of the lensed system and nearby objects using the Subaru telescope. To be able to detect any faint objects near the quasar, the Subaru resolution and sensitivity is combined with the technique that can effectively remove the light from the bright quasar images. We also present new observations of the lensed system with the $1.3 \mathrm{~m}$ telescope at the CTIO observatory in the $J, H$, and $K$ bands. Based on these NIR and our previous optical observations of UM673, we obtain new estimates for some of the observational constraints. Thus, we analyse the extinction properties of the lensing galaxy and present new measurements of the extinction-corrected flux ratio between the A and B components of UM673. We discuss possible models of the lens constrained by the measured observables (the image positions, flux ratio, and time delay). Unlike previous studies of UM673, we use the time delay to constrain the model of the lens. The nearby lens perturber, if any, is likely to be a very faint object that is difficult to detect with high significance. Therefore, when analysing the Subaru data, the time-delay information is incorporated to search for the probable location of the perturber.

The paper is organised as follows: the results of the photometric analysis of the Subaru and CTIO data are given in Sects. 3 and 4 . In Sect. 5 we examine extinction properties of the lensing galaxy. The model of the lens that can reproduce the positions of the UM673 components, their flux ratio, and the time delay is discussed in Sect. 6. We conclude with a summary and discussion given in Sect. 7.

\section{Observations}

The Subaru observations were carried out with the Infra-red Camera and Spectrograph (IRCS; Kobayashi et al. 2000) using an AO system with 188 control elements (AO188; Hayan et al. 2010) in the natural guide star (NGS) mode. UM673 was observed in the $H$ band on February 1, 2012. The images were taken with a $1024 \times 1024$ ALADDIN III CCD. The pixel scale was $20 \mu$ as, resulting in a field of view (FOV) of $21^{\prime \prime}$. The gain of the CCD was $5.6 \mathrm{e}^{-}$/ADU. Imaging was performed using the non-destructive readout scheme with 16 readouts that reduced the readout noise by a factor of $1 / \sqrt{16}$. The resulting readout noise was estimated to be 2.03 ADU. As the FOV of UM673 does not contain nearby bright stars (within $1^{\prime}$ of UM673), the brighter component $\mathrm{A}\left(m_{\mathrm{R}} \sim 16.5 \mathrm{mag}\right)$ of the lensed system was selected as a guide star. The night of the observations was very clear with natural seeing of about $0.5^{\prime \prime}$ in the $K$ band. UM673 was observed at five dither positions shifted by $9^{\prime \prime}$. Every dither sequence was repeated five times. The total number of images taken was 25 ( 22 of them were used in the following analysis). The exposure time of each image was $150 \mathrm{~s}$. Seeing of the images varied from $0.28^{\prime \prime}$ to $0.46^{\prime \prime}$. The airmass of UM673 varied from 1.35 to 1.88 . We also observed nearby standard star Feige $16\left(m_{\mathrm{H}}=12.336 \mathrm{mag}\right)$ from the MKO JHK Catalogue (Leggett et al. 2006) with and without the AO correction. In both cases the star was observed in one five-points dither sequence. The exposure time of the observations without the AO correction was $20 \mathrm{~s}$, while the exposure time with the AO correction was $5 \mathrm{~s}$. The airmass and full width at half maximum (FWHM) of the star in the observations with the correction were 1.85 and $0.2^{\prime \prime}$, while without the correction they were 1.18 and $0.6^{\prime \prime}$.

The near-infrared (NIR) observations of UM673 with the 1.3 m SMARTS telescope (CTIO, Chile) were carried out for a total of 23 nights between August 16, 2009 and January 18, 2010 in the $J, H$, and $K$ bands. These data were acquired as a part of the ToO observations carried out by National Central University, Taiwan. The $1.3 \mathrm{~m}$ SMARTS telescope is equipped with the dual-channel optical/NIR CCD camera ANDICAM which has a NIR FOV of about $2.2^{\prime} \times 2.2^{\prime}$ and a pixel scale of $0.297^{\prime \prime}$ pixel $^{-1}$. On each observational night images were taken in a series of four frames in the $J, H$, and $K$ bands, simultaneously with the optical data in the $V, R$, and $I$ bands (see Koptelova et al. 2012). The exposure time of the images in the $J, H$, and $K$ bands varied from 132 to $225 \mathrm{~s}$.

\section{Photometry of the AO Subaru data}

The Subaru $H$-band image frames of UM673 were reduced with the IRAF $^{1}$ scripts written for the reduction of the IRCS data by Minowa $^{2}$ (2010; see also Minowa et al. 2005). The bad pixels were removed by interpolation from adjacent good pixels using file cam_badpix.coo ${ }^{3}$ with the positions of the IRCS bad pixels. We corrected the images for the relative pixel offset and coadded them into a stack image using median-filter coaddition.

The $17^{\prime \prime} \times 10^{\prime \prime}$ subregion of the stack image is shown in Fig. 1. The FWHM of the stack image estimated from component A of UM673 is about $0.43^{\prime \prime}$. A lower left-corner window displays the light from the lensing galaxy after subtracting quasar images A and B (see Sect. 3 for details). Figure 2 shows the exposure map of the stack image presented in Fig. 1. The white central region of the map with the marked locations of quasar images $\mathrm{A}$ and $\mathrm{B}$ corresponds to a maximum exposure time of $55 \mathrm{~min}$.

To calibrate the fluxes, we measured a zero-magnitude using the combined (stack) images of the standard star observed with the AO correction, as in this case the airmass of the star

1 IRAF is distributed by the National Optical Astronomy Observatories, which are operated by the Association of Universities for Research in Astronomy, Inc., under cooperative agreement with the National Science Foundation.

2 The scripts can be found at the Subaru Data Reduction webpage http: //www . naoj .org/Observing/DataReduction/index.html 3 This file is available at the Subaru IRCS webpage http://www . naoj .org/Observing/Instruments/IRCS/ 


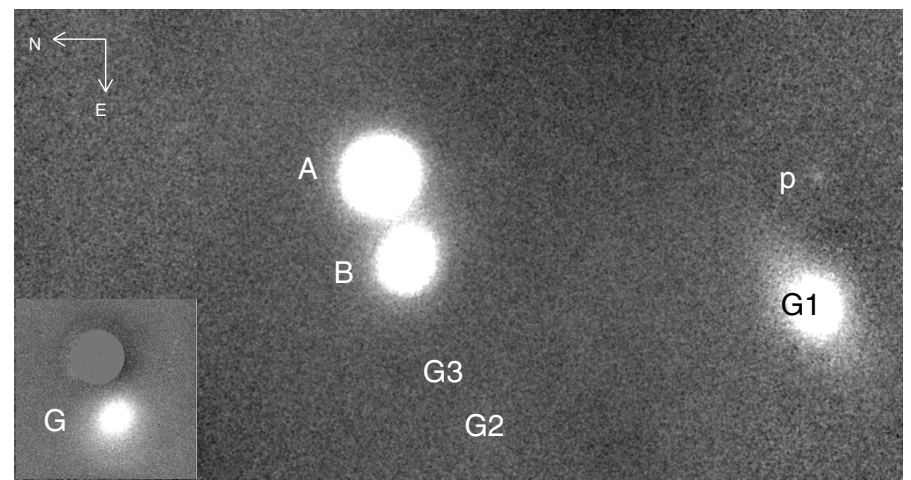

Fig. 1. 17" $\times 10^{\prime \prime}$ Subaru H-band stack image of UM673. Lensing galaxy $\mathrm{G}$ after subtracting the light from quasar images $\mathrm{A}$ and $\mathrm{B}$ is shown in a lower zoom window.

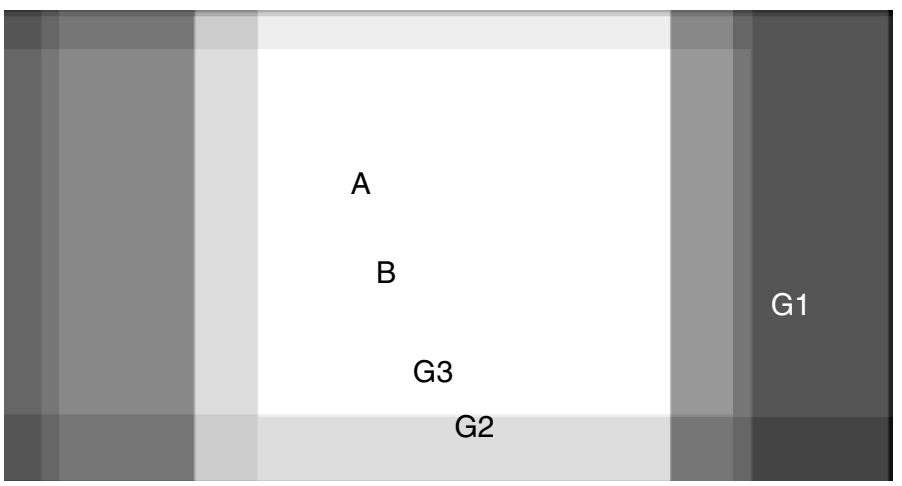

Fig. 2. Exposure map of the UM673 field presented in Fig. 1. The grey scale corresponds to the total integration time.

and UM673 was almost the same. The $H$-band zero-magnitude was determined to be $24.294 \pm 0.013 \mathrm{mag}$ for $1 \mathrm{ADU}$ per second. We also estimated a limiting magnitude for the point source detection in the combined image of UM673 using component $\mathrm{A}$ of the quasar. The $5 \sigma$ limiting magnitude measured as in Minowa (2010) was estimated to be about 22.5 mag at an aperture radius of $0.3^{\prime \prime}$. The $5 \sigma$ surface brightness limiting magnitude for the region of the combined image with the longest total exposure was found to be about $21.6 \mathrm{mag} \operatorname{arcsec}^{-2}$. The $5 \sigma$ surface brightness limiting magnitude with bright field galaxy G1 was estimated to be about $20.5 \mathrm{mag} \operatorname{arcsec}^{-2}$.

To detect possible faint objects in the field of UM673 we ran SExtractor (Bertin \& Arnouts 1996) on the stack image. The SExtractor search parameters were fixed to those used by Minowa et al. (2005) for the faint-source detection in the deep NIR observations with the Subaru telescope. The SExtractor did not detect any faint objects except for a low-level signal at position $\mathrm{p}$ (see Fig. 1). This signal results from the persistent afterimage of quasar component $\mathrm{A}$ and at first was misclassified by us as a new faint galaxy. The image persistence of component $\mathrm{A}$ was noticed in every fourth image of the five-image dither sequence. In other dither images the effect was weaker or not present at all. Fig. 1 also shows the locations of the two faint galaxies G2 and G3 detected in the HST observations of UM673 (Lehár et al. 2000). The H-band magnitudes of these galaxies as measured by Lehár et al. (2000) are about 20.65 and 21.78 mag. Their locations correspond to the longest integration time in the Subaru stack image (see Fig. 2). However, we detected no objects at the HST positions of G2 and G3 as they appear to be fainter than the limiting magnitude achieved in the Subaru observations.

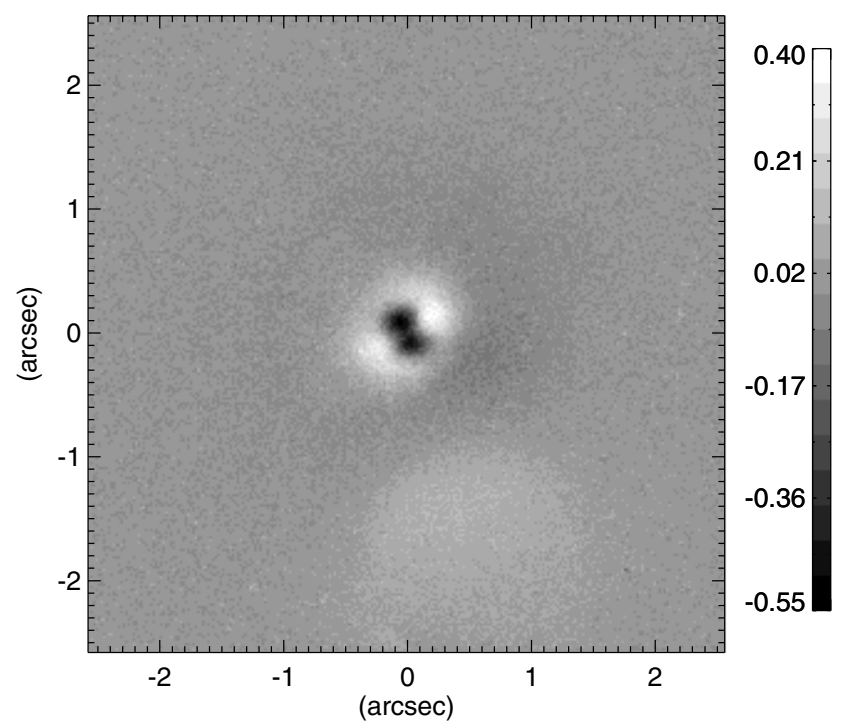

Fig. 3. Residuals after subtracting the PSF model constructed using observations of the standard star.

The photometric analysis of the lensed system was performed with the point spread function (PSF) fitting method. The model of the PSF was constructed using component A of UM673. To analyse the brightness distribution of the PSF, we extracted a 5" subframe from the UM673 frame centred on component A. The light from component B in the subframe was blocked using a circular mask. The PSF model was constructed in two steps. First, the PSF was modelled as a combination of the elliptical Gaussian and Moffat (Moffat 1969) profiles. In these calculations we used the brightness distribution of the standard star to include all hard-to-model features of the AO PSF into the PSF model. We blurred the star PSF with the combined Gaussian-Moffat profile, so that the resulting PSF was fitted to the brightness distribution of component A. The residuals between the data and the model are shown in Fig. 3. The vertical bar demonstrates pixel counts in ADU s ${ }^{-1}$ that can be compared with a sky background sigma of $0.018 \mathrm{ADU} \mathrm{s}^{-1}$. As can be seen, this PSF model cannot describe the shape of the observed PSF in its centre. Therefore, second, we replaced the central region (within a radius of $0.7^{\prime \prime}$ ) of the best-fit Gaussian-Moffat PSF with the observed brightness distribution of component $\mathrm{A}$. This hybrid analytical-and-empirical PSF was used for the photometric analysis of the UM673 lensed system.

The brightness profile of each of the quasar components was modelled as the Dirac delta function convolved with the analytical-and-empirical PSF. We approximated the delta function using a narrow Gaussian with a sigma of 0.5 pixels $\left(0.01^{\prime \prime}\right)$. The model parameters describing the quasar components are the fluxes of components A and B scaled with the PSF and their $x$ and $y$ coordinates on the frame.

The galaxy brightness distribution was modelled with the Sérsic profile of the form

$I(r)=I_{\mathrm{e}} \exp \left\{-b_{\mathrm{n}}\left[\left(\frac{r}{r_{\mathrm{e}}}\right)^{\frac{1}{n}}-1\right]\right\}$,

where $I_{\mathrm{e}}$ is the intensity at effective radius $r_{\mathrm{e}}$ (Sérsic 1968); $r^{2}=x^{2}+y^{2} / q^{2}$ is specified by parameter $q$, the axis ratio of the ellipse. Quantity $b_{\mathrm{n}}$ is a function of shape parameter $n$ and defined so that $r_{\mathrm{e}}$ encloses half of the total light (see Ciotti 1991). In the calculations we express $I_{\mathrm{e}}$ in Eq. (1) through the total 

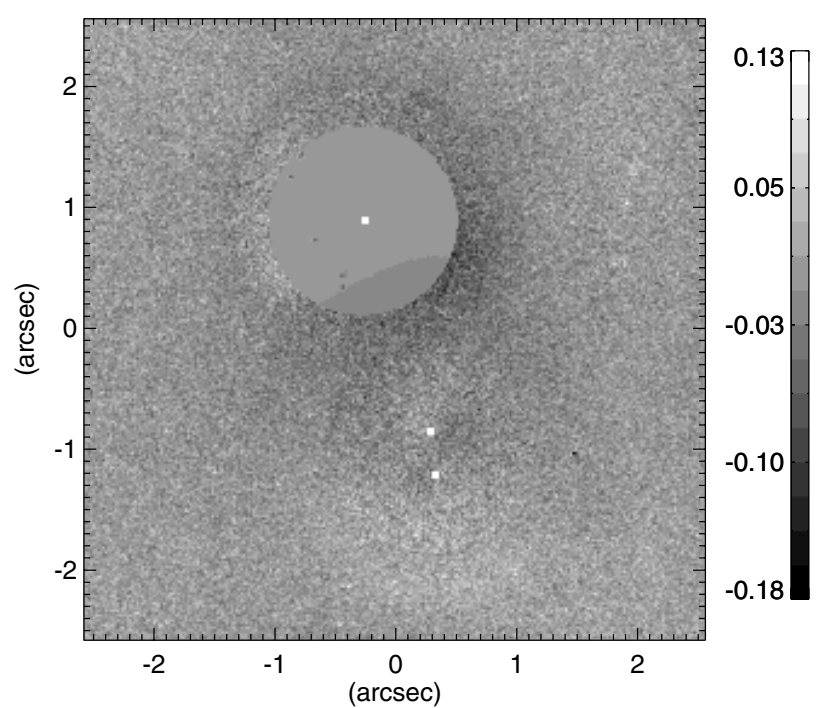

Fig. 4. Residuals after subtracting the light of quasar images A and B, and the lensing galaxy. The positions of A, B, and the galaxy centre as calculated from the model are shown by white dots.

Table 1. Relative astrometry of component B, the lens, and the nearby bright galaxy.

\begin{tabular}{lcccc}
\hline \hline ID & $z$ & RA(") & $\operatorname{Dec}\left({ }^{\prime \prime}\right)$ & $H(\mathrm{mag})$ \\
\hline A & 2.72 & 0 & 0 & $15.174 \pm 0.015$ \\
B & 2.72 & $2.101 \pm 0.004$ & $-0.585 \pm 0.005$ & $17.335 \pm 0.018$ \\
G & 0.49 & $1.751 \pm 0.015$ & $-0.540 \pm 0.005$ & $17.816 \pm 0.022$ \\
G1 & 0.17 & $3.028 \pm 0.004$ & $-9.929 \pm 0.004$ & $17.854 \pm 0.015$ \\
\hline
\end{tabular}

Table 2. Galaxy parameters: Sérsic index $n$; effective radius $r_{\mathrm{e}}$; axis ratio $q$; and position angle $\mathrm{PA}$.

\begin{tabular}{lcccc}
\hline \hline ID & $n$ & $r_{\mathrm{e}}\left({ }^{\prime \prime}\right)$ & $q=\frac{b}{a}$ & $\mathrm{PA}\left({ }^{\circ}\right)$ \\
\hline G & $3.93 \pm 0.06$ & $0.86 \pm 0.08$ & $0.80 \pm 0.02$ & $30.5 \pm 3.4$ \\
G1 & $4.28 \pm 0.39$ & $0.85 \pm 0.09$ & $0.68 \pm 0.03$ & $125.9 \pm 2.2$ \\
\hline
\end{tabular}

luminosity $L_{\text {tot }}$ (see also Eq. (2) in Ciotti 1991 and Eq. (2) in Graham \& Driver 2005). Thus, the Sérsic profile is described with the following free parameters: centre coordinates of the galaxy $x_{\mathrm{c}}$ and $y_{\mathrm{c}}, L_{\mathrm{tot}}, r_{\mathrm{e}}, n, q$, and position angle PA of the galaxy major axis. The Sérsic model of the galaxy was then convolved with the PSF.

The solution for the best-fit photometric model of UM673 $\left(\chi_{v}^{2} \sim 0.003\right)$ is summarised in Tables 1 and 2. (Note that we used minimum change in $\chi_{v}^{2}$ as the stopping criterion for the minimisation procedure.) Table 1 presents the positions of component $\mathrm{B}$ and the lensing galaxy relative to component $\mathrm{A}$, and the calibrated magnitudes of the quasar components and lensing galaxy. The tables also present the results of the fit of galaxy G1 with the Sérsic profile and its Sérsic magnitude. The magnitude errors in Table 1 are the errors of the fit, which also include the error of the zero-magnitude. Figure 4 shows residuals between the observational data and the model. In this figure a circular region at the location of component A corresponds to the central part of the hybrid PSF described empirically. The lensing galaxy after subtracting the light from quasar components A and B is shown in the lower left corner of Fig. 1.

With the goal to identify any faint object at small impact parameters from the quasar, we also considered another approach. We subtracted the light from the quasar using the principal

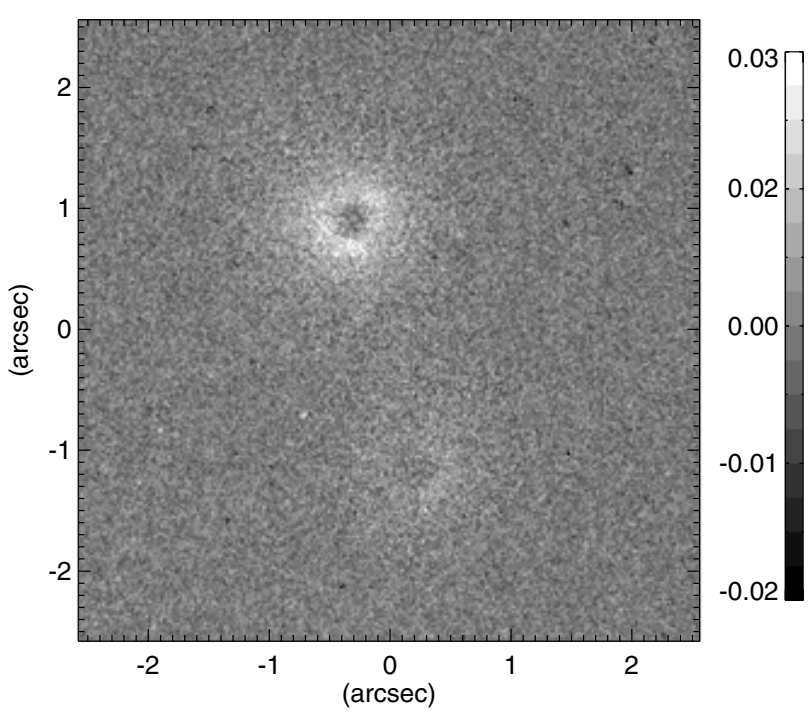

Fig. 5. Residuals after subtracting the scaled PC modes from the stack image.

component (PC) representation of the AO complex PSF as described in Chun et al. $(2006,2010)$. The PC decomposition was applied to a sequence of the single-exposure subframes. We analysed a sequence of 22 subframes of UM673 that contained both components A and B. In each subframe, component A represents the best model for the PSF. The PCs were calculated by diagonalising the covariance matrix between the 22 singleexposure PSFs. The resulting PSF $P(x, y)$ in the combined image is expressed as a linear combination of the PC components, $P(x, y)=\sum_{i=1}^{22} a_{i} \mathrm{PC}_{i}(x, y)$, where $a_{i}$ are the coefficients that represent the importance of each of the PC modes of the AO PSF in the combined image and quantify the variations of the AO PSF with time. Then, the model, with $a_{i}$ as parameters, was fitted to the observational data.

The residuals between the observational data and the PC model are shown in Fig. 5. As can be seen in the figure, the PSF constructed using the PC decomposition is able to remove most of the light from the quasar, especially in the central region. The overall scatter in the residuals appears to be small with higher values at the location of component $\mathrm{A}$ and lower values at the location of component B. In this residual map we do not detect any statistically significant signal (above $1 \sigma$ detection) that might be produced by a field object. However, we caution that some of the individual images have irregular sky background that contributes to the sky background of the stack image. We found that these irregularities in the sky (especially small-scale irregularities) can lead to mildly significant detections (below $1 \sigma$ ) in the residual map. As most of these detections usually disappear after more careful background correction, we considered them false detections. If there were a real object with low significance, it would be hard to distinguish its signal from these background variations.

\section{Photometry of the CTIO data}

The CTIO NIR data of UM673 were reduced in a similar way as the Subaru data. We created sky flat-fields in the $J, H$, and $K$ bands using target frames taken at slightly offset positions. The target frames were dark subtracted and divided by the normalised sky flat-fields. The reduced frames taken on the same nights were aligned and co-added. 
Table 3. Positions and NIR magnitudes of the calibration stars.

\begin{tabular}{lccccc}
\hline \hline ID & RA & Dec & $J(\mathrm{mag})$ & $H(\mathrm{mag})$ & $K(\mathrm{mag})$ \\
\hline$\alpha$ & $1^{\mathrm{h}} 45^{\mathrm{m}} 16.55^{\mathrm{s}}$ & $-9^{\circ} 46^{\prime} 29.54^{\prime \prime}$ & $16.823 \pm 0.159$ & $16.280 \pm 0.225$ & 16.106 \\
$\beta$ & $1^{\mathrm{h}} 45^{\mathrm{m}} 15.83^{\mathrm{s}}$ & $-9^{\circ} 45^{\prime} 43.44^{\prime \prime}$ & $17.216 \pm 0.015$ & $16.683 \pm 0.054$ & $16.165 \pm 0.075$ \\
\hline
\end{tabular}

The FOV of the CTIO NIR images contains only one star, about 30" south-west of UM673. We denote this star as $\beta$. We used star $\beta$ to construct the PSF and to calibrate fluxes. The fluxes of the quasar components relative to the star were measured following the procedure described in Koptelova et al. (2008, 2010, 2012). Star $\beta$ itself does not have measured calibrated magnitudes in the $J, H$, and $K$ bands. To calibrate the flux of star $\beta$ we used the available archive NIR observations of UM673 obtained with the VLT in July $2000^{4}$. The FOV of the VLT images is larger $\left(2.5^{\prime} \times 2.5^{\prime}\right)$ and includes more stars than the CTIO images. One of the bright stars in the VLT images of UM673 has the measured 2MASS $J, H$ and $K$-band magnitudes (Skrutskie et al. 2006). The position of this star (denoted as $\alpha$ ) and its 2MASS magnitudes are given in Table 3 . We calibrated the flux of $\beta$ relative to the flux of $\alpha$ (see Table 3 ). Finally, we calibrated the fluxes of components A and B of UM673 relative to $\operatorname{star} \beta$.

The NIR brightness of the lensing galaxy located closer to component B of UM673 is similar to the brightness of B (see Table 1 in Sect. 3 and Lehár et al. 2000). Therefore, we expect a significant contribution of the galaxy flux to the flux of $\mathrm{B}$ in the CTIO NIR data. This contribution cannot be estimated based on the CTIO images alone, as their spatial resolution does not allow for separation of the fluxes of component B and the lensing galaxy. We estimated the galaxy flux contamination of B in the CTIO measurements using the VLT NIR observations of $\mathrm{UM} 673^{4}$, which are deeper and have higher angular resolution $\left(0.147^{\prime \prime}\right.$ pixel $\left.^{-1}\right)$. From the VLT data we measured the fluxes of $\mathrm{A}$ and B corrected for the galaxy flux. In these measurements the galaxy brightness was modelled with the de Vaucouleurs profile (de Vaucouleurs 1948). Then, the VLT data were rescaled and blurred, so as to match the CTIO data in the spatial resolution and typical seeing. After that, the fluxes of components A and B were measured again but without the correction for the galaxy flux. From the comparison of the fluxes of the quasar components measured in these two ways (with and without the galaxy flux correction) we find that, within errors of measurements, the galaxy does not contribute to the flux of component A. But its contribution to the flux of component B is significant and estimated to be $0.663,0.836$, and $0.999 \mathrm{mag}$ in the $J, H$, and $K$ bands. These values were taken into account in the CTIO photometry of component $\mathrm{B}$.

The measured $J$ - and $H$-band magnitudes of the quasar components are given in Tables 4 and 5. We could not apply the PSF photometry to the $K$-band data. The signal from UM673 and the PSF star in the CTIO $K$-band images was very low as the $K$ band suffered a very high background level. Therefore, we instead measured the average $K$-band flux ratio between the quasar components. In these measurements, the PSF was constructed using component A of UM673. The $K$-band flux ratio averaged over the observations taken between September 30 and October 28, 2009 is estimated to be $2.151 \pm 0.040 \mathrm{mag}$. The $J$ and $H$-band flux ratios averaged over the period of the observations are $2.183 \pm 0.006$ and $2.162 \pm 0.010$.

\footnotetext{
4 PI/CoI J. Hjorth et al. Proposal No. 65.O-0666(D).
}

Table 4. Photometry of components A and B of UM673 in the $J$ band.

\begin{tabular}{cccc}
\hline \hline JD & Seeing( $\left(^{\prime \prime}\right)$ & $J_{\mathrm{A}}(\mathrm{mag})$ & $J_{\mathrm{B}}(\mathrm{mag})$ \\
\hline 2455063.801 & 1.8 & $15.356 \pm 0.013$ & $17.535 \pm 0.021$ \\
2455064.825 & 1.3 & $15.362 \pm 0.005$ & $17.541 \pm 0.006$ \\
2455069.816 & 1.4 & $15.407 \pm 0.016$ & $17.555 \pm 0.023$ \\
2455070.769 & 1.4 & $15.382 \pm 0.023$ & $17.569 \pm 0.029$ \\
2455116.652 & 1.3 & $15.395 \pm 0.006$ & $17.604 \pm 0.013$ \\
\hline
\end{tabular}

Table 5. Photometry of components A and B of UM673 in the $H$ band.

\begin{tabular}{cccc}
\hline \hline JD & Seeing(") & $H_{\mathrm{A}}(\mathrm{mag})$ & $H_{\mathrm{B}}(\mathrm{mag})$ \\
\hline 2455070.792 & 1.4 & $15.210 \pm 0.012$ & $17.372 \pm 0.016$ \\
2455082.758 & 1.4 & $15.229 \pm 0.006$ & $17.380 \pm 0.028$ \\
2455083.796 & 1.2 & $15.218 \pm 0.013$ & $17.375 \pm 0.015$ \\
2455090.789 & 1.3 & $15.182 \pm 0.018$ & $17.359 \pm 0.027$ \\
2455095.750 & 1.5 & $15.189 \pm 0.014$ & $17.370 \pm 0.024$ \\
2455100.658 & 1.3 & $15.181 \pm 0.016$ & $17.376 \pm 0.038$ \\
2455105.730 & 1.6 & $15.202 \pm 0.009$ & $17.377 \pm 0.029$ \\
2455116.675 & 1.3 & $15.186 \pm 0.012$ & $17.342 \pm 0.021$ \\
\hline
\end{tabular}

The employed procedure of measuring the uncontaminated flux of B based on the VLT data is simple and accurate at the same time. However, we note that there are other approaches as well. Ricci et al.(2013) adopted an alternative way of estimating the galaxy flux contribution to B in their photometry of the optical UM673 data obtained with the Danish $1.54 \mathrm{~m}$ telescope. In the method the galaxy flux is constrained using the HST positions and photometry.

\section{Intrinsic flux ratio between A and B}

The observed flux ratio between the quasar components might differ from the flux ratio produced by lensing on the galaxy. We refer to the flux ratio resulting from lensing as the intrinsic flux ratio. Given that the fluxes of the quasar components are corrected for the time delay, the intrinsic flux ratio can be altered by microlensing on the stars in the lens, by dust extinction in the lensing galaxy or other objects between the quasar and observer. We assume that the observed fluxes of A and B differ from their intrinsic fluxes mainly because of the dust extinction, as there was no clear microlensing detected in the previous observations of UM673 (see Wisotzki et al. 2004; Koptelova et al. 2008, 2010, 2012). We also explain the microlensing-like behaviour (the bluer-when-brighter) of component A found by Nakos et al. (2005) by the quasar intrinsic variability and not by microlensing (see also the discussion in Koptelova et al. 2012).

The amount of dust reddening at a given wavelength can be estimated from the observed multi-band flux ratios of the quasar components by adopting a certain extinction law in the lensing galaxy. For this purpose we used the $V, R, I, J, H$ and $K$-band flux ratios between components A and B of UM673. The optical flux ratios are taken from the measurements of Koptelova et al. (2012), while the NIR flux ratios are those from the CTIO observations presented in Sect. 4. The flux ratios in the optical bands were averaged over several epochs to eliminate the 
effect of the time delay on the single-epoch measurements. As shown in Koptelova et al. (2012), the multi-epoch average flux ratio between components $\mathrm{A}$ and $\mathrm{B}$ is a good approximation for their time-delay-corrected flux ratio. The NIR light curves are too short to be corrected for the time delay. In the calculation of the NIR flux ratios, we assumed that the effect of the time delay is weak and within the photometric errors. This is a valid assumption as quasar brightness variations in the NIR bands are smaller than in the optical bands, and UM673 itself was very quiet during our NIR observations.

We also used the $B$-band flux ratio measured based on different single-epoch observations of UM673. The average $B$-band flux ratio was calculated based on our own observations of UM673 with the $1.3 \mathrm{~m}$ CTIO telescope and the measurements published earlier by Elíasdóttir et al. (2006) and Mosquera et al. (2011). The CTIO B-band flux ratio between A and B was estimated using the observations obtained on August 26, September 30, and October 11, 2009. It was measured to be $2.246 \pm 0.008 \mathrm{mag}$. The multi-epoch weighted average flux ratio adopted by us for the calculations is $2.320 \pm 0.025 \mathrm{mag}$.

The $J, H$ and $K$-band flux ratios were corrected for the galaxy contribution as described in Sect. 4 of this paper. The galaxy contribution to the optical fluxes is difficult to estimate based on the low-resolution ground-based observations alone. Here, we estimated the galaxy contribution in the optical bands using the following strategy: it is known from observations of UM673 that the galaxy flux contribution to component $\mathrm{B}$ increases from the $B$ band, in which it is negligible (we can likely assume it to be zero), to the $K$ band, in which it becomes much more significant (about $1 \mathrm{mag}$ ). The contamination of component B by the galaxy light was also noticed in the spectroscopic observations of Surdej et al. (1988), who found an excess in the continuum radiation of the component at $\lambda>5800 \AA$ (this lower wavelength roughly corresponds to the $V$ band) due to the galaxy light contribution. Therefore, the galaxy contamination of component B, if not taken into account, introduces a certain trend into the flux ratio-wavelength dependance. We estimated this trend using the $B, J, H$ and $K$-band measurements of the galaxy flux contribution to component $\mathrm{B}$. The dependence of the contribution on wavelength was fitted with a second-order polynomial. From the resulting curve we estimated the amount of the galaxy flux contribution to each band between the $B$ and $K$ bands. Then the optical flux ratios were corrected for this amount by subtracting the second-order polynomial from the observed flux ratios, measured without the galaxy correction.

From the analysis we find that the galaxy contribution to the flux of component B is about $0.098,0.204$, and 0.363 mag in the $V, R$, and $I$ bands. The corrected flux ratios at the optical wavelengths and the measured NIR flux ratios are shown in Fig. 6 by circles. The $H$-band flux ratio measured from the Subaru observations of UM673 is marked by a triangle. As can be seen, the Subaru flux ratio measured based on the single-epoch observations agrees well with the CTIO $H$-band flux ratio measured based on the multi-epoch data. The resulting flux ratios in the $B, V, R, I, J, H$, and $K$ bands were used to measure the differential dust extinction between the lines of sights to components A and $\mathrm{B}$ and also to estimate their intrinsic flux ratio.

We analysed the extinction properties of the lensing galaxy using the NIR extinction law (see review of Mathis 1990 and references therein) and the parametrisation of the Galactic extinction law given in Cardelli et al. (1989). The magnitude difference $\Delta m^{\mathrm{BA}}(\lambda)$ and the flux ratio between the quasar components are related as $\Delta m^{\mathrm{BA}}=2.5 * \lg \left(f_{\mathrm{A}} / f_{\mathrm{B}}\right)$. The intrinsic magnitude difference between the quasar components is defined as

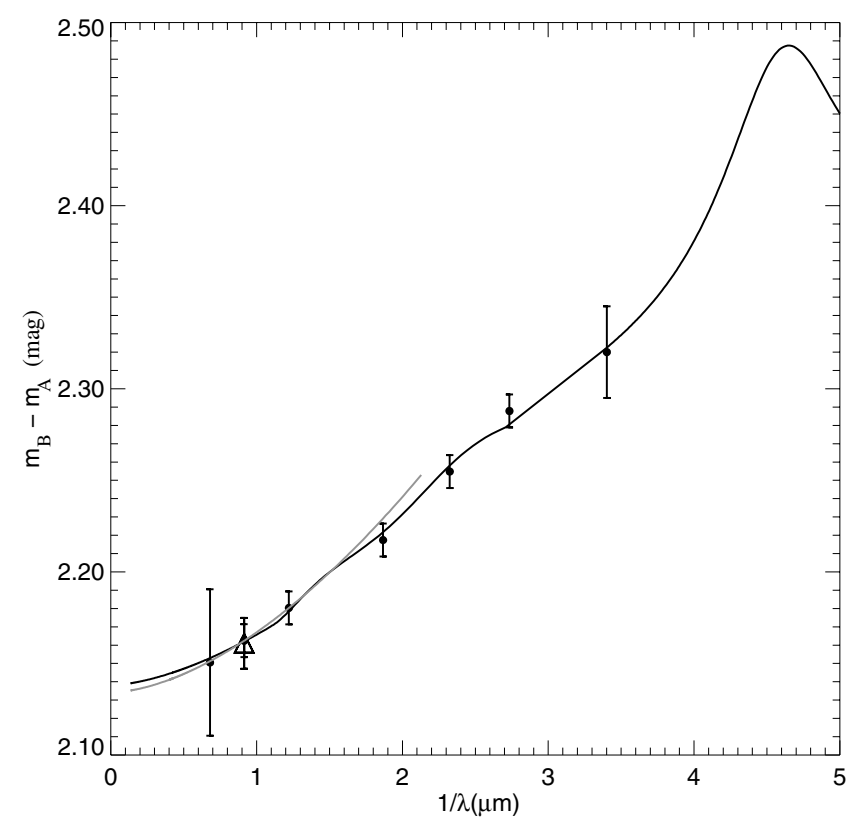

Fig. 6. Magnitude difference between components A and B of UM673 as a function of the rest-frame wavelength. The fit to the NIR measurements of the magnitude differences is shown by a grey line, while the fit to the optical-NIR measurements is shown by a black line.

$\Delta m_{0}^{\mathrm{BA}}=m_{0}^{\mathrm{B}}(\lambda)-m_{0}^{\mathrm{A}}(\lambda)$ and does not depend on wavelength. The differential extinction at wavelength $\lambda$ is determined by the discrepancy between the observed and intrinsic magnitude differences as $\Delta A(\lambda)=\Delta m^{\mathrm{BA}}(\lambda)-\Delta m_{0}^{\mathrm{BA}}$. Generally, this differential extinction accounts for all possible causes of reddening of the quasar components, dust reddening in the lensing galaxy, and in the intervening objects along the line of sight to each of the components.

The NIR magnitude difference between components A and $\mathrm{B}$ is represented as follows:

$\Delta m^{\mathrm{BA}}(\lambda)=\Delta m_{0}^{\mathrm{BA}}+\Delta A(J)\left(\frac{\lambda}{1.25 \mu \mathrm{m}}\right)^{-1.7}$,

with $\triangle A(J)=A^{\mathrm{B}}(J)-A^{\mathrm{A}}(J)$ (see Mathis 1990). The model defined by Eq. (2) has two parameters, $\Delta m_{0}^{\mathrm{BA}}$ and $\triangle A(J)$. From the fit, we calculated $\Delta m_{0}^{\mathrm{BA}}=2.134 \pm 0.024 \mathrm{mag}$ and $\Delta A(J)=$ $0.023 \pm 0.014 \mathrm{mag}\left(\chi_{v}^{2} \sim 0.0\right)$. The differential extinction in the $H$ and $K$ bands was estimated to be about 0.015 and 0.009 mag.

For the extinction curve parameterised as in Cardelli et al. (1989), the magnitude difference between components A and $\mathrm{B}$ is determined by the intrinsic magnitude difference $\Delta m_{0}^{\mathrm{BA}}$, the differential extinction $\triangle A(V)$, and the differential colour excess $\Delta E(B-V)$ as follows:

$\Delta m^{\mathrm{BA}}(\lambda)=\Delta m_{0}^{\mathrm{BA}}+\Delta A(V) a\left(\lambda^{-1}\right)+\Delta E(B-V) b\left(\lambda^{-1}\right)$,

where $\Delta A(V)=A^{\mathrm{B}}(V)-A^{\mathrm{A}}(V)$ and $\Delta E(B-V)=E^{\mathrm{B}}(B-V)-$ $E^{\mathrm{A}}(B-V)$. From the fit between the observed and model magnitude differences, we calculated $\Delta m_{0}^{\mathrm{BA}}=2.138 \pm 0.015 \mathrm{mag}$, $\Delta A(V)=0.081 \pm 0.018 \mathrm{mag}$, and $\Delta E(B-V)=0.035 \pm$ $0.003 \operatorname{mag}\left(\chi_{v}^{2} \sim 0.3\right)$.

Thus, the values of $\Delta m_{0}^{\mathrm{BA}}$ derived from the NIR and Galactic extinction curves agree well. The effective ratio of the total-toselective extinction from the data is $\Delta A(V) / \Delta E(B-V) \simeq 2.29$. This value is in the range of the $R_{V}$ values measured by Patil et al. (2007) for a sample of 26 early-type galaxies. 


\section{UM673 lens model}

In this section we explore lens models for the UM673 system. We analysed the models to identify the source of shear and to estimate the probable location of the nearby perturber. The models were constrained using the positions of the two quasar components, their flux ratio, and a time delay of $89 \pm 11$ days measured in Koptelova et al. (2012). In the calculations we used positions of the quasar components and properties of the lensing galaxy as measured from the Subaru images of UM673 (see Tables 1 and 2). The adopted value of $H_{0}$ is $74.2 \pm 3.6 \mathrm{~km} \mathrm{~s}^{-1} \mathrm{Mpc}^{-1}$ as measured in Riess et al. (2009)

The lensing galaxy was modelled as a singular isothermal ellipsoid (SIE; see Kassiola \& Kovner 1993; Kormann et al. 1994; Keeton \& Kochanek 1998). The properties of the SIE model and corresponding gravitational potential are given in Keeton \& Kochanek (1998) and Keeton (2001). The model parameters were calculated by minimising the following function:

$\chi^{2}=\chi_{\text {posn }}^{2}+\chi_{\text {flux }}^{2}+\chi_{\text {delay }}^{2}$

where $\chi_{\text {posn }}^{2}$ is the $\chi^{2}$ term for the image positions; $\chi_{\text {flux }}^{2}$ is the $\chi^{2}$ term for the image flux ratio, and $\chi_{\text {delay }}^{2}$ is the $\chi^{2}$ term for the time delay. In our calculations the $\chi_{\text {posn }}^{2}$ term was evaluated in the source plane as described in Keeton (2010). The $\chi_{\text {flux }}^{2}$ term is defined as $\chi_{\text {flux }}^{2}=\left(f_{\mathrm{B}} / f_{\mathrm{A}}-\mu_{\mathrm{B}} / \mu_{\mathrm{A}}\right)^{2} / \sigma_{\text {flux }}^{2}$, where $f_{\mathrm{B}} / f_{\mathrm{A}}$ and $\mu_{\mathrm{B}} / \mu_{\mathrm{A}}$ are the observed and model flux ratio. The $\chi_{\text {delay }}^{2}$ term was calculated as $\chi_{\text {delay }}^{2}=\left(\Delta t_{\mathrm{BA}}^{\mathrm{obs}}-\Delta t_{\mathrm{BA}}^{\mathrm{mod}}\right)^{2} / \sigma_{\text {delay }}^{2}$. The models were fitted using our routines written in the interactive data language (IDL).

First, following Keeton et al. (1998) and Lehár et al. (2000), we considered the SIE lens model with an external shear (SIE $+\gamma$, see Kochanek 1991; Bernstein \& Fischer 1999; Keeton 2001; Schneider et al. 2006). We denote this model as Model 1. The introduction of the external shear is justified by the presence of the nearby galaxies detected in the deep HST and Subaru images of UM673. The locations of the nearby objects are illustrated in the scheme presented in Fig. 7. In the figure, the major axis of the lensing galaxy is aligned with the horizontal axis. The scheme shows the orientation of the total external shear as measured in Lehár et al. (2000) from all objects within 20" of UM673 (marked by a dashed grey line). The direction to the largest external shear is shown by a solid grey line (see Table 4 in Lehár et al. 2000). Model 1 has the following free parameters: mass of the lens $b$, axis ratio $q$ and position angle PA of the galaxy, shear strength $\gamma$ and its orientation $\theta_{\gamma}$, and actual position of the quasar. The galaxy ellipticity and position angle are chosen to be free parameters because models with fixed $q$ and PA do not fit well ( $\chi^{2}>100$, see also Lehár et al. 2000).

Table 6 summarises the results of the fit. The solution for the Model 1 parameters calculated using Eq. (4) is given in the second column of the table. The critical lines for this solution are shown in the left panel of Fig. 8. The values of the model parameters presented in the first column of Table 6 were calculated for the SIE $+\gamma$ model constrained only by the image positions and the flux ratio $\left(\chi^{2}=\chi_{\text {posn }}^{2}+\chi_{\text {flux }}^{2}\right)$. The errors on the model parameters were obtained from the corresponding covariance matrix calculated at the last iteration of the minimisation process.

As can be seen in Table 6, the position angle of the lensing galaxy in Model 1 is different from the angle of the observed galaxy light distribution (see Table 2). The shear is oriented almost along the line that connects components A and B. There are two faint galaxies, G2 and G3, detected in the HST images of UM673 along this direction (see Fig. 7). Therefore, from

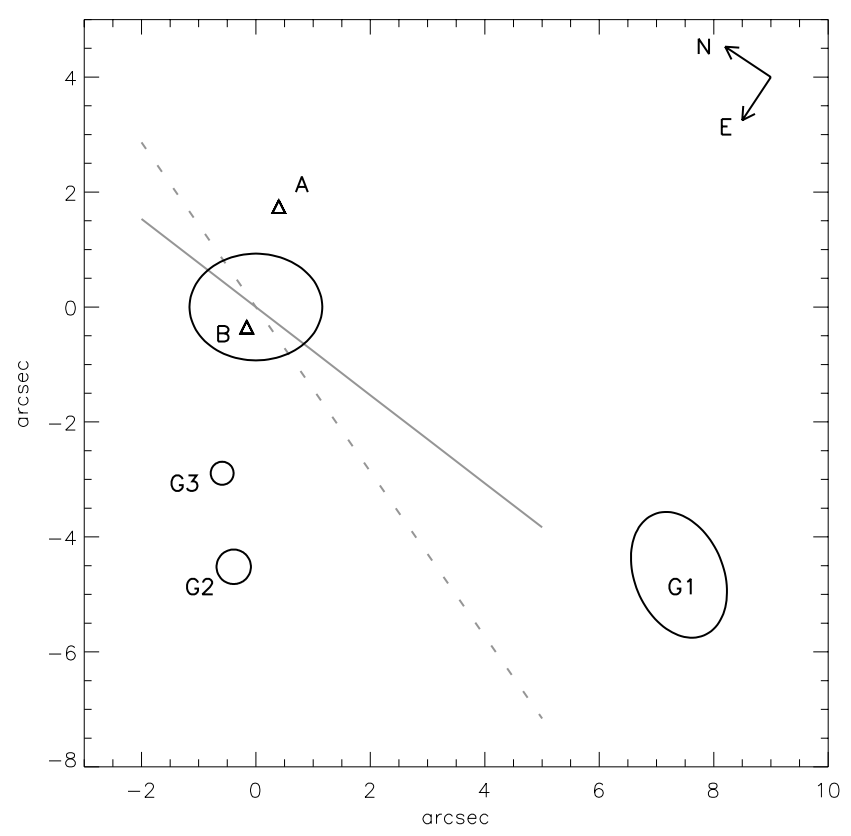

Fig. 7. Locations of the nearby objects in the vicinity of UM673. The galaxies are shown with their critical curves as if they were isolated objects. The orientations of the total and the largest shear in the field are shown by dotted and solid grey lines.

Table 6. Model 1 results: the SIE model with an external shear.

\begin{tabular}{ccc}
\hline \hline Parameter & SIE $+\gamma$ & SIE $+\gamma$ \\
\hline$b\left(^{\prime \prime}\right)$ & $1.155 \pm 0.009$ & $1.157 \pm 0.009$ \\
$q$ & $0.792 \pm 0.004$ & $0.784 \pm 0.003$ \\
$\mathrm{PA}\left({ }^{\circ}\right)$ & $56.7 \pm 0.5$ & $57.1 \pm 0.3$ \\
$\gamma$ & $0.075 \pm 0.003$ & $0.068 \pm 0.003$ \\
$\theta_{\gamma}\left({ }^{\circ}\right)$ & $53 \pm 2$ & $51 \pm 2$ \\
\hline$\mu_{\mathrm{B}} / \mu_{\mathrm{A}}$ & 0.140 & 0.141 \\
$\Delta t_{\mathrm{BA}}($ days $)$ & 115 & 114 \\
\hline$\chi^{2} / N_{\text {D.o.F. }}$ & $0.0 /-2$ & $5.3 /-1$ \\
\hline
\end{tabular}

Model 1 we conclude that the mass towards G2 and G3 probably influences the orientation and ellipticity of the lensing potential in the model.

We also considered a lens model with galaxy parameters $q$ and PA fixed to the values measured from the observations. In this model, we assumed that G3, which is closer to the main lens, has a stronger effect on its potential. The mass concentration towards G3 was modelled as a singular isothermal sphere (SIS) with one free parameter, mass $b_{\mathrm{p}}$. The lensing effect from other nearby galaxies at the position of the lens was described by the external shear parameters. We denote this model as Model 2. The solution for Model 2 is summarised in the first column of Table 7. The critical lines for the model are shown in the middle panel of Fig. 8. In Model 2, the G3 perturber has a moderate mass and there is also a source of shear at an angle of about $50^{\circ}$ measured counterclockwise from the galaxy major axis.

In both Model 1 and Model 2, the mass at the locations of G2 and G3 has no effect on the time delay. To examine which model of the lens might lead to the observed delay of about 89 days, we considered a model with a source of shear at the location of galaxy G3 and an arbitrarily located SIS perturber of unknown mass $b_{\mathrm{p}}$ (labelled $G_{\mathrm{p}}$ ). We denote this model as Model 3. The model has seven parameters and therefore is 

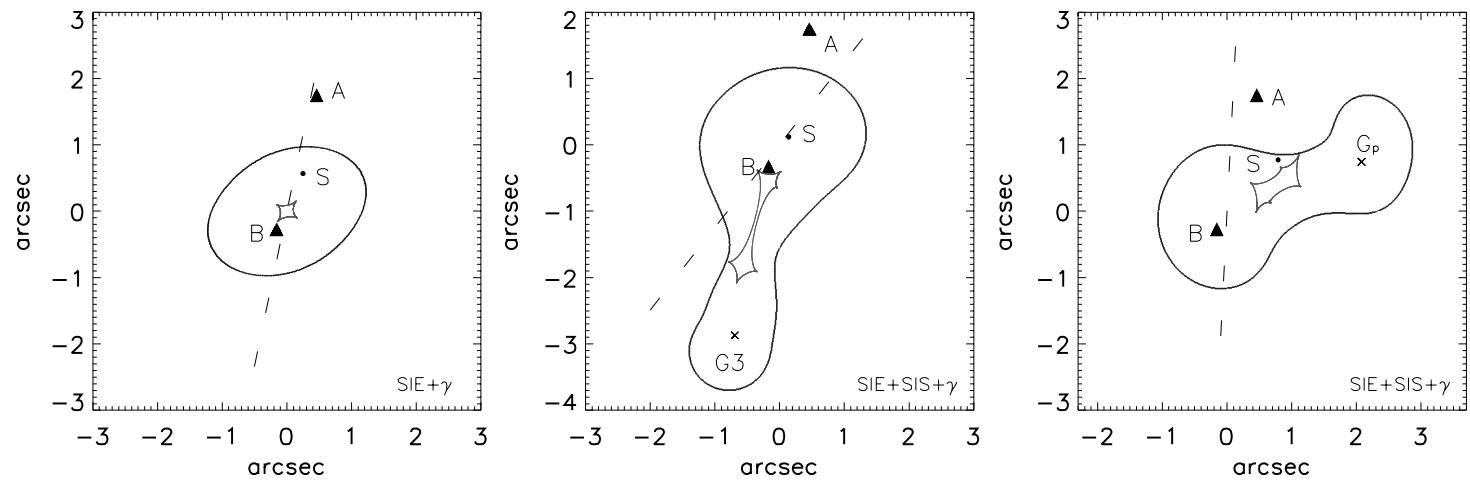

Fig. 8. Critical curves (black) and caustics (grey) for the lens models presented in Tables 6 and 7 (right: Model 1, middle: Model 2, left: Model 3). The positions of quasar images A and B are shown by triangles. The source position is marked by a circle. Dashed lines depict the orientation of the external shear. Crosses indicate the positions of the perturbers.
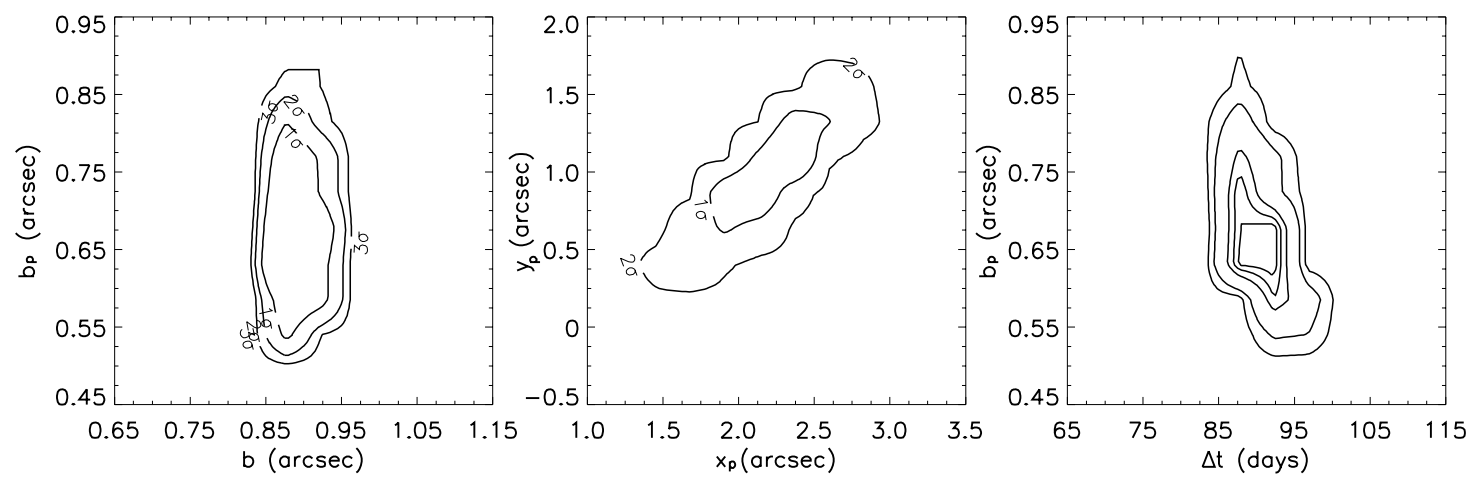

Fig. 9. Contours of the joint probability distribution in the plane of lens mass $b$ and perturber mass $b_{\mathrm{p}}$ (left); in the plane of the $x_{\mathrm{p}}$ and $y_{\mathrm{p}}$ perturber coordinates relative to the main lens (middle); in the plane of time delay $\Delta t$ and perturber mass $b_{\mathrm{p}}($ right).

Table 7. Model 2 and 3 results: the SIE-and-SIS model with an external shear.

\begin{tabular}{ccc}
\hline \hline Parameter & SIE + SIS $+\gamma(2)$ & SIE + SIS $+\gamma(3)$ \\
\hline$b\left(^{\prime \prime}\right)$ & $1.091 \pm 0.014$ & $0.910_{-0.011}^{+0.036}$ \\
$b_{\mathrm{p}}\left({ }^{\prime \prime}\right)$ & $0.601 \pm 0.010$ & $0.648_{-0.087}^{+0.146}$ \\
$x_{\mathrm{p}}\left({ }^{\prime \prime}\right)$ & - & $2.080_{-0.280}^{+0.420}$ \\
$y_{\mathrm{p}}\left(^{\prime \prime}\right)$ & - & $0.745_{-0.197}^{+0.478}$ \\
$\gamma$ & $0.042 \pm 0.003$ & $0.173_{-0.009}^{+0.024}$ \\
$\theta_{\gamma}\left({ }^{\circ}\right)$ & $51 \pm 2$ & $87_{-10}^{+1}$ \\
\hline$\mu_{\mathrm{B}} / \mu_{\mathrm{A}}$ & 0.141 & 0.140 \\
$\Delta t_{\mathrm{BA}}($ days $)$ & 116 & 90 \\
\hline$\chi^{2} / N_{\text {D.O.F. }}$ & $6.0 / 0$ & $0.1 /-2$ \\
\hline
\end{tabular}

underconstrained. Two of the parameters, the shear strength and its orientation, can be estimated. We estimated the shear strength to be $\gamma=b_{\mathrm{G} 3} / 2 r_{\mathrm{G} 3}$, where $b_{\mathrm{G} 3}$ and $r_{\mathrm{G} 3}$ are the mass and distance of G3 from the main lens. The shear angle was assumed to be equal to the observed angle towards G3 within an uncertainty of $10^{\circ}$. From the fit we find that in this model the mass of the main lens is smaller than in Model 1. The presence of the SIS perturber of a moderate mass near the lens also makes the total mass between A and B less concentrated. Model 3 predicts similar positions of the quasar images and their flux ratio as the previous two models. At the same time, its mass configuration favours shorter time delays.

To assess dependencies between the parameters in Model 3 and estimate the parameter uncertainties we performed a statistical analysis of the solutions. We minimised the $\chi^{2}$ function for 500 realisations of Model 3. Each time we added Gaussian distributed errors to the input shear strength and its orientation. We also added the Gaussian error to the position of the SIS perturber relative to the lensing galaxy. In this analysis, the probable location of the SIS perturber was constrained by only considering the solutions that favour time delays shorter than 100 days. (In the configurations with longer delays, perturber mass $b_{\mathrm{p}}$ becomes unimportant, and the configurations closely resemble Models 1 and 2.)

Figure 9 illustrates the result of the analysis. The two first panels of the figure show contours corresponding to the $1 \sigma, 2 \sigma$, and $3 \sigma$ confidence intervals in the plane of two parameters: mass of the main lens $b$ and mass of the perturber $b_{\mathrm{p}}$; coordinates $x_{\mathrm{p}}$ and $y_{\mathrm{p}}$ of the perturber, respectively. (The $3 \sigma$ contour in the middle panel is not shown because it is only poorly resolved.) As can be seen in Fig. 9, the mass of the main lens in the parameter realisations of Model 3 is localised in a narrow range of $0.9-1.0^{\prime \prime}$. At the same time, the range of the possible masses of the perturber is wider because $b_{\mathrm{p}}$ depends on the position of the perturber relative to the main lens. The models with the larger distance between the lens and perturber predict a higher mass of the perturber. As can be seen in the right panel of Fig. 9, which shows contours of the equal probability distribution in the plane of the time delay and perturber mass, the delay is shorter for the perturber with the higher mass. But at the same time, the delay can also be shorter in the models with the pertuber with the smaller mass located closer to the main lens. The most probable values of the Model 3 parameters and their $1 \sigma$ uncertainties are summarised in Table 7 . The corresponding critical lines for Model 3 are shown in the right panel of Fig. 8. 
In Models 2 and 3, perturber $G_{\mathrm{p}}$ is assumed to be located in the plane of the main lensing galaxy. To test the model predictions for the case when $z_{\mathrm{G}_{\mathrm{p}}}>z_{1}$, we also analysed the two-plane lens model reviewed in Schneider et al. (1992). In this analysis we set the SIS perturber at different redshifts $z_{\mathrm{G}_{\mathrm{p}}}>z_{1}$ and adopted the best solution of Model 3 as an initial guess for the model parameters. As in Model 3, the external shear was associated with galaxy G3. From the calculations we find that the impact parameter of the perturber from the quasar line of sight becomes smaller as its redshift increases. These configurations produce a noticeable lensing effect (see also the discussion in Keeton 2003; and Momcheva et al. 2006). For the case when $z_{\mathrm{G}_{\mathrm{p}}}=1.63$ (the redshift of the DLA absorber), we adopted one additional constraint assuming that the position of quasar image A or, alternatively, quasar image B in the perturber's plane is close to the position of $G_{\mathrm{p}}$ within a conservative uncertainty of $0.1^{\prime \prime}$. The better solution $\left(\chi^{2} / N_{\text {D.O.F. }} \simeq 13 / 0\right)$ corresponds to the configuration when $G_{\mathrm{p}}$ is closer to image B in the perturber's plane. However, this model probably forms the third image of the quasar because the predicted position of $G_{\mathrm{p}}$ in this model is very close to the quasar line of sight $\left(\sim 0.15^{\prime \prime}\right)$. The formation of the third image can probably be suppressed if we assume a density profile shallower than the isothermal model for the perturber (e.g., the NFW density profile, Navarro et al. 1996). The other possibility is that the perturber is located at a redshift lower than $z_{\mathrm{G}_{\mathrm{p}}}=1.63$ and, correspondingly, at the larger impact parameter from the quasar line of sight.

\section{Summary and discussion}

We presented new high-resolution AO observations of the double-lensed quasar UM673 obtained with the Subaru telescope in the $H$ band. Based on the Subaru data, we measured optical properties of the lensing galaxy and the nearby bright galaxy in the field of UM673. The measured $H$-band magnitudes of the galaxies (17.816 and $17.854 \mathrm{mag}$, respectively) agree well with the magnitudes previously estimated based on the HST data (Lehár et al. 2000).

We also presented results of the new NIR observations of UM673 obtained with the $1.3 \mathrm{~m}$ SMARTS telescope at the CTIO observatory in 2009. Based on these observational data, we measured weighted $J, H$ and $K$-band magnitude differences between the UM673 components of about $2.180 \pm 0.006,2.162 \pm 0.009$, and $2.151 \pm 0.040 \mathrm{mag}$. The flux ratios between components $\mathrm{A}$ and B estimated from the analysis of the archive VLT $J, H$ and $K$-band images of UM673 are $2.270 \pm 0.044,2.216 \pm 0.057$, and $2.187 \pm 0.012 \mathrm{mag}$.

From the comparison of the CTIO, VLT, HST NIR flux ratios and the Gemini NIR flux ratio presented in Fadely \& Keeton (2011) we found a difference of up to 0.1 mag between different measurements. The older observations (2000 and earlier) with the VLT and especially with the HST result in higher estimates of the NIR flux ratios than the more recent CTIO and Subaru observations. This disagreement cannot be fully explained by the differences in the photometric procedure. (In our test analysis we measured the same HST $H$-band flux ratio as Lehár et al. 2000.) As there were no detailed NIR observations of UM673 before 2000 to explain this discrepancy, we connected this difference with the possible evolution of the flux ratio with time (for example because of quasar variability), although systematic errors in the estimation of the flux ratio cannot be entirely excluded.

We analysed the dependence of the flux ratio on wavelength based on recent extensive multi-band observations (see Koptelova et al. 2008, 2010, 2012; Ricci et al. 2013; Oscoz et al. 2013) and our new NIR observations of UM673. From this analysis we measured the extinction properties of the lensing galaxy and the intrinsic flux ratio between the UM673 components produced by lensing on the galaxy. We estimated the effective ratio of the total-to-selective extinction in the $V$ band to be $\simeq 2.29$. This value agrees well with the much more precise measurement of $R_{\mathrm{V}}$ in the early-type lensing galaxy of the system SBS 0909+532 (see Motta et al. 2002). It also agrees well with the results of Patil et al. (2007), who studied extinction properties of early-type galaxies and measured $R_{V}$ to be in a range of 2.03-3.46 with an average of 3.02. The intrinsic flux ratio between the UM673 components was estimated to be $2.138 \pm 0.015 \mathrm{mag}$. This value agrees better with the spectroscopic observations of Wisotzki et al. (2004) than the intrinsic flux ratio previously estimated from the HST observations of UM673 in Falco et al. (1999).

The time delay between the two UM673 components as measured by Koptelova et al. (2012) and Oscoz et al. (2013) based on different datasets is somewhat shorter than the delay predicted by the single-lens model. The shorter delay leads to a value of the Hubble parameter that is higher than the results of other independent measurements (e.g., Riess et al. 2009; Planck Collaboration XVI 2014). This indicates a possible additional lensing effect from nearby objects or objects on the line of sight to the quasar (see, e.g., Keeton et al. 2000; Keeton \& Zabludoff 2004). Lehár et al. (2000) have measured a total convergence of $k_{\mathrm{p}}=0.138$ from the nearby objects in the field of UM673 based on the previous HST observations. However, this value cannot account for the shorter time delay and might be underestimated. The total external convergence can probably be measured with a better accuracy, e.g., by adopting the larger radius around UM673 for the analysis of the external tidal effects or by more detailed imaging of the UM673 nearby field.

We performed new detailed imaging of the UM673 assuming that the additional mass is associated with a high-redshift lineof-sight object at a small impact parameter from the quasar (such as the host galaxy of the DLA absorber discovered by Cooke et al. 2010). Imaging of the high- $z$ DLA host galaxy requires long integration times at the wavelength of the DLA emission as well as special techniques that allow for removing the quasar light contamination. In the current analysis of the 55-min Subaru $H$-band image of the lensed quasar, we applied the principal component representation of the complex AO PSF to subtract the light from the UM673 quasar components. We did not convincingly detect any faint objects within $5^{\prime \prime}$ of UM673 in the residual image.

Analysis of the imaging data was accompanied by the model analysis of the lens. From the modelling we identified the source of shear and estimated the probable location of nearby perturbing object $G_{\mathrm{p}}$, the DLA host galaxy candidate. The shear is probably associated with the field galaxies G2 and G3 seen in the HST observations of UM673, while the observed time delay can only be reproduced by the model with an additional perturbing mass between components A and B (Model 3). The inferred total mass of the lensing galaxy and the perturber is consistent with the mass of the lens constrained by the positions of the UM673 components alone. This is attributed to the degeneracy between the mass of the lens and the external convergence (Falco et al. 1985; Gorenstein et al. 1988; Bernstein \& Fischer 1999). The use of the time delay as an additional constraint helped to distinguish between the mass of the lens and the mass of the perturber, but there is still a degeneracy between the perturber mass and its distance from the lens. For the case when $z_{\mathrm{G}_{\mathrm{p}}}>z_{1}$ 
the higher-redshift perturber is probably located at the smaller impact parameter from the lensing galaxy (and also from the quasar) and so affects its lensing properties.

More conclusions about the UM673 lens model require additional observations. In particular, the measurement of the velocity dispersion of the lens galaxy will tightly constrain its mass; the detailed analysis of the lens environment will help to reduce errors in the lens model. A more accurate measurement of the time delay between the UM673 components will also improve the mass model of the lens.

Acknowledgements. We thank the referee for useful comments and help in improving the content of this paper. We would like to thank Luis Goicoechea for interesting discussions on the topic. We thank the PhD student H.H. Chan for his efforts in testing the NFW lens model based on the data presented in this paper. We appreciate the Subaru time allocation committee for scheduling our observations (proposal ID: S12A0150S; PI: E. Koptelova). The research project was supported by the National Taiwan University grant No. 10R40044 and by the Taiwan National Science Councils grant No. NSC99-2811-M-002-051. This research has made use of the ESO Science Archive data taken with the VLT telescope.

\section{References}

Bernstein, G., \& Fischer, P. 1999, ApJ, 118, 14

Bertin, E., \& Arnouts, S. 1996, A\&AS, 117, 393

Cardelli, J. A., Clayton, G. C., \& Mathis, J. S. 1989, ApJ, 345, 245

Ciotti, L. 1991, A\&A, 249, 99

Chun, M. R., Gharanfoli, S., Kulkarni, V. P., \& Takamiya, M. 2006, AJ, 131, 686

Chun, M. R., Kulkarni, V. P., Gharanfoli, S., \& Takamiya, M. 2010, AJ, 139, 296

Cooke, R., Pettini, M., Steidel, C. C., et al. 2010, MNRAS, 409, 679

de Vaucouleurs, G. 1948, Ann. Astrophys., 11, 247

Eigenbrod, A., Courbin, F., \& Meylan, G. 2007, A\&A, 465, 51

Elíasdóttir, Á., Hjorth, J., Toft, S., Burud, I., \& Paraficz, D. 2006, ApJS, 166, 443

Fadely, R., \& Keeton, C. R. 2011, AJ, 141, 101

Falco, E. E., Gorenstein, M. V., \& Shapiro, I. I. 1985, ApJ, 289, L1

Falco, E. E., Impey, C. D., Kochanek, C. S., et al. 1999, ApJ, 523, 617

Fassnacht, C. D., \& Lubin, L. M. 2002, AJ, 123, 627

Fassnacht, C. D., Gal, R. R., Lubin, L. M., et al. 2006, ApJ, 642, 30

Faure, C., Alloin, D., Kneib, J. P., \& Courbin, F. 2004, A\&A, 428, 741

Goicoechea, L. J., \& Shalyapin, V. N. 2010, ApJ, 708, 995

Gorenstein, M. V., Shapiro, I. I., \& Falco, E. E. 1988, ApJ, 327, 693

Graham, A. W., \& Driver, S. P. 2005, PASA, 22, 118

Hayano, Y. Takami, H., Oya, S., et al. 2010, Proc. SPIE, 7736, 8

Kassiola, A., \& Kovner, I. 1993, ApJ, 417, 459

Keeton, C. R. 2001 [arXiv: astro-ph/0102341]

Keeton, C. R. 2003, ApJ, 584, 664

Keeton, C. R. 2010, Gen. Relativ. Gravit., 42, 2151

Keeton, C. R., \& Kochanek, C. S. 1998, ApJ, 495, 157

Keeton, C. R., \& Moustakas, L. A. 2008, ApJ, 699, 1720

Keeton, C. R., \& Zabludoff, A. I. 2004, ApJ, 612, 660

Keeton, C. R., Kochanek, C. S., \& Falco, E. E. 1998, ApJ, 509, 561
Keeton, C. R., Christlein, D., \& Zabludoff, A. I. 2000, ApJ, 545, 129

Kneib, J.-P., Alloin, D., \& Pello, R. 1998, A\&A, 339, L65

Kobayashi, N., Tokunaga, A. T., Terada, H., et al. 2000, Proc. SPIE, 4008, 1056 Kochanek, C. S. 1991, ApJ, 382, 58

Koptelova, E., Artamonov, B. P., Bruevich, V. V., Burkhonov, O. A., \& Sergeev, A. V. 2008, Astron. Rep., 52, 270

Koptelova, E., Oknyanskij, V. L., Artamonov B. P., \& Burkhonov, O. 2010, MNRAS, 401, 2805

Koptelova, E., Chen, W. P., Chiueh, T., et al. 2012, A\&A, 544, A51

Kormann, R., Schneider, P., \& Bartelmann, M. 1994, A\&A, 284, 285

Leggett, S. K., Currie, M. J., Varricatt, W. P., et al. 2006, MNRAS, 373, 781

Lehár, J., Falco, E. E., Kochanek, C. S., et al. 2000, ApJ, 536, 584

MacLeod, C. L., Kochanek, C. S., \& Agol, E. 2009, ApJ, 699, 1578

Mathis, J. S. 1990, Annu. Rev. Astron. Astrophys., 28, 37

Minowa, Y. 2010, in Adaptive Optics Systems II, eds. B. L. Ellerbrock, M. Hart, N. Hubin, \& P. L. Wizionwich, Proc. SPIE, 7736, 122

Minowa, Y., Kobayashi, N., Yoshii, Y., et al. 2005, ApJ, 629, 29

Moffat, A. F. J. 1969, A\&A, 3, 455

Møller, P., Warren, S. J., Fall, S. M., Fynbo, J. U., \& Jakobsen, P. 2002, ApJ, 574,51

Momcheva, I., Williams, K., Keeton, C., \& Zabludoff, A. 2006, ApJ, 641, 169

Mosquera, A. M., Muñoz, J. A., Mediavilla, E., \& Kochanek, C. S. 2011, ApJ, 728,145

Motta, V., Mediavilla, E., Muñoz, J. A., et al. 2002, ApJ, 574, 719

Nakos, Th., Courbin, F., Poels, J., et al. 2005, A\&A, 441, 443

Navarro, J. F., Frenk, C. S., \& White, S. D. M. 1996, ApJ, 462, 563

Oscoz, A., Serra-Ricart, M., Mediavilla, E., \& Muñoz, J. A. 2013, ApJ, 779, 144

Patil, M. K., Pandey, S. K., Sahu, D. K., \& Kembhavi, A. 2007, A\&A, 461, 103

Péroux, C., Bouché, N., Kulkarni, V. P., York, D. G., \& Vladilo, G. 2011, MNRAS, 410, 2237

Planck Collaboration XVI. 2014, A\&A, in press, DOI: 10.1051/0004-6361/201321591

Rauch, M., Sargent, W. L. W., Barlow, T. A. 2001, ApJ, 554, 823

Refsdal, S. 1964, MNRAS, 128, 307

Ricci, D., Elyiv, A., Finet, F., et al. 2013, A\&A, 551, A104

Riess, A. G., Macri, L., Casertano, S., et al. 2009, ApJ, 699, 539

Sérsic J. L. 1968, Atlas de Galaxias Australes (Cordoba: Observatorio Astronomico, Argentina)

Schneider P., Ehlers J., \& Falco E. E. 1992, Gravitational Lenses (New York: Springer), 281

Schneider, P., Kochanek, C., \& Wambsganss, J. 2006, Gravitatational lensing: strong, weak and micro Saas-Free Advanced Cource 33, eds. G. Meylan, P. Jetzer, \& P. North (Springer: Netherlands), 125

Skrutskie, M. F., Cutri, R. M., Stiening, R., et al. 2006, AJ, 131, 1163

Smette, A., Surdej, J., Shaver, P. A., et al. 1992, ApJ, 389, 39

Surdej, J., Magain, Swings, J.-P., et al. 1987, Nature, 329, 695

Surdej, J., Magain, Swings, J.-P., et al. 1988, A\&A, 198, 49

Tonry, J. L., \& Kochanek, C. S. 2000, AJ, 119, 1078

Treu, T., Gavazzi, R., Gorecki, A., et al. 2009 ApJ, 690, 670

Wambsganss, J., Bode, P., \& Ostriker, J. P. 2005, ApJ, 635, L1

Williams, K. A., Momcheva, I., Keeton, C. R., Zabludoff, A. I., \& Lehár, J. 2006, ApJ, 646, 85

Wisotzki, L., Becker, T., Christensen, L., et al. 2004, Astron. Nachr., 325, 135

Wolfe, A. M., Turnshek, D. A., Smith, H. E., \& Cohen, R. D. 1986, ApJS, 61, 249 